\title{
Association of Diabetic Retinopathy with Angiographic Severity of Coronary Artery Disease in Patients with Non- ST Elevation Myocardial Infarction
}

\author{
HERU AL AMIN¹, AMAL KUMAR CHOUDHURY'1, SYED ALI AHSAN², BIJOY DUTTA ${ }^{1}$, MUJTAHID \\ MOHAMMAD HOSSAIN ${ }^{1}$, NUR ALAM ${ }^{1}$, KOFIL UDDIN ${ }^{1}$, PARTHA PRATIM SAHA ${ }^{1}$ \\ ${ }^{1}$ Department of Cardiology, National Institute of Cardiovascular Disease \& Hospital (NICVD), Dhaka, Bangladesh \\ ${ }^{2}$ Department of Cardiology,Bangabandhu Sheikh Mujib Medical University, Shahbag, Dhaka, Bangladesh \\ Address of Correspondence: Dr. Heru Al Amin, Department of Cardiology, National Institute of Cardiovascular Disease \& Hospital (NICVD), \\ Dhaka, Bangladesh, E-mail: hirualaminrpmc@gmail.com
}

\begin{abstract}
Background: Bed side ophthalmoscopic examination is a simple measure of diagnosis of diabetic retinopathy and has been shown to be a predictor of poor outcome in various cardiovascular conditions including coronary artery disease (CAD). Retinopathy lesions are fairly common findings in clinic settings and may predict risk of coronary heart disease (CHD). The present study was intended to find the relationship between diabetic retinopathy with the severity of coronary artery disease in patients with NSTEMI.
\end{abstract}

Methods: This cross-sectional observational study was conducted in the Department of cardiology, National Institute of Cardiovascular Diseases and Hospital (NICVD), Dhaka, Bangladesh, from March 2019 to August 2020. A total of 120 DM with NSTEMI patients undergoing coronary angiogram and also fundoscopic examination with fundal photography during the index hospitalization were included in this study. Study subjects were divided into two groups on the basis of diabetic retinopathy (Group-I: NSTEMI with diabetic retinopathy ; Group- II: NSTEMI without diabetic retinopathy). Severity of coronary artery disease was determined by Gensini score and correlation between diabetic retinopathy and Gensini score was assessed.

Results: Gensini score was significantly higher in patients with diabetic retinopathy than that in patients without diabetic retinopathy (62.2 \pm 27.7 vs. $43.3 \pm 25.3, p<0.001)$. Gensini score increased with increasing severity of diabetic retinopathy $(P<0.001)$.The risk of having severe $C A D$ in patient with diabetic retinopathy was $13.03(95 \% C I=2.410-70.419)(P<0.003)$. A significant correlation between diabetic retinopathy and Gensini score was noted (p value $<0.001)$

Conclusion: It may be concluded that presence and severity of diabetic retinopathy is associated with angiographically severe coronary artery disease in patient with non-ST elevation myocardial infarction (NSTEMI) and it may be considered as an independent predictor of severity of CAD. As is a bed side assessment, so before performing coronary angiography, it appears to be additive for risk stratification.

Keywords: Coronary artery disease, Diabetic retinopathy, Left ventricular ejection fraction, Gensini score TVD, RCA, LC

University Heart Journal 2022; 18(1): 50-53

\section{Introduction:}

Cardiovascular diseases (CVDs) are the most common cause of premature death in the world. CVDs account for $50 \%$ of all non-communicable disease (NCD) deaths in the world each year and represent a significant threat to human welfare and sustainable development. ${ }^{1}$ Coronary artery disease (CAD) is an increasingly important medical and public health problem and is the leading cause of mortality in Bangladesh. Like other, South Asians Bangladeshis are unduly prone to develop CAD which is often premature in onset, follows a rapidly progressive course and angiographically more sever. The exact prevalence of CAD in Bangladesh is not known. Only a limited number of small-scale epidemiological studies are available. More recent data indicates that $\mathrm{CAD}$ prevalence is $1.85 \%$ to $3.4 \%$ in rural population and $19.6 \%$ in an urban population. ${ }^{2}$

Diabetes Mellitus is a heterogeneous primary disorder of carbohydrate metabolism with absolute insulin deficiency (Type 1) or relative insulin deficiency (type 2), resistance or both leading to hyperglycemia.3 The South Asian region shares a major proportion of this worldwide burden of 
diabetes. The prevalence of diabetes ranges from $0.9 \%$ in Bangladesh to $21.2 \%$ in india.

CVDs were the primary cause of Diabetes mellitus is a vascular disease with many microvascular manifestation as retinopathy and macro vascular complication as coronary artery disease. Unstable angina patient with DR have more significant left ventricular dysfunction than without DR (El Demerdash et al., 2012). To date, no studies have focused on the relation between DR and the extent and severity of CAD. Therefore, the aim of the present study was to evaluate the relationship between the presence of DR and indices of the extent and severity of CAD detected by coronary angiography.

\section{Methods}

Study patients: One hundred twenty cases were selected from consecutive type 2 DM patients with NSTEMI who underwent coronary angiography between March 2019 to August 2020. Type 2 DM was diagnosed according to the recommendations of the American Diabetes Association. Exclusion criteria were known duration of DM of less than 1 year, type 1 diabetes mellitus, uncontrolled hypertension (systolic blood pressure $>180 \mathrm{mmHg}$ and/or diastolic blood pressure $>100 \mathrm{mmHg}$ ), previous coronary bypass surgery, and known nondiabetic retinal disease.

Coronary angiography: Selective coronary cineangiography was performed from the radial or femoral approach using Judkin's technique. Multiple views were obtained in all patients, with the left anterior descending and left circumflex coronary arteries visualized in at least four views and the right coronary artery in at least two views by using cine-angiographic equipment. Coronary angiograms were scored according to followings-

Severity score: This was the Gensini score, which has been described previously. Briefly, the coronary arterial tree was divided into segments with multiplying factors according to the functional importance of any given segment ( 5 for the left main trunk to 0.5 for the most distal segments) and the percent reduction in lumen diameter of each narrowing was assigned a score $(0,1,2,4,8,16,32$ according to the degree of stenosis). The sum of the scores of all segments gives the Gensini score, which places emphasis on the severity of the disease.

Detection of DR: Fundoscopic examination was done one day after admission. Initially tropicamide $1.0 \%$ eye drop was given in both eyes. After full dilatation of both pupils fundoscopic examination was done by Keeller ophthalmoscope for detection of diabetic retinopathy. Then next day fundal photo was done and assessed by an ophthalmologist. patient was divided into two groups on the basis of presence or absence of diabetic retinopathy. Those with presence of retinopathy categorized as group I and absence of retinopathy as group II. We also evaluated risk factors for CAD, which included patient age, sex, duration of diabetes, hypertension, hypercholesterolemia, smoking status, history of a cerebrovascular accident or peripheral arterial disease, and family history of CAD. Subjects who had quit smoking for over 5 years were considered nonsmokers. The therapeutic modality for DM (oral antidiabetics or insulin) and history of previous myocardial infarction (MI) were also recorded. Biochemical parameters including blood fasting glucose, serum total cholesterol, triglycerides, high-density lipoprotein (HDL), low-density lipoprotein (LDL), creatinine were noted.

Statistics: Results are expressed as the mean \pm SD. For univariate analysis, the significance of differences between the two groups for continuous variables was assessed with the unpaired Student's $t$ test, while the chi-square test was used for nominal variables. Comparisons of the severity of CAD with severity of diabetic retinopathy were performed with 2-way ANOVA using appropriate covariates (with parameters having significant differences in univariate analysis). The correlations between the CAD severity with other parameters were analyzed using simple correlation analysis. Multivariate associations of the CAD severity and extent scores were determined by performing multiple stepwise linear regression analysis with parameters having significant correlations in the simple correlation analysis. Statistical analyses were carried out by using SPSS 16.0 (Statistical Package for the Social Sciences by SPSS Inc., Chicago, IL, USA).

\section{Results}

Diabetic retinopathy: According to the results of the fundus examinations, $60(50 \%)$ of 120 patients had DR (non proliferative mild in 16, moderate to severe in 22 , and proliferative in 22) whereas the remaining $60(50 \%)$ had no retinopathy.

Comparison of clinical characteristics and risk factors: The patients with DR had a significantly longer duration of known diabetes $(P<0.001)$, a higher ratio of being on insulin therapy $(P=0.03)$ in table 4.3 , higher serum creatinine levels $(P<0.001)$ in table 4.5. There were no significant differences with regard to patient age, sex, risk factors for CAD, and other biochemical parameters (Table I). The differences in the severity of CAD persisted even after multivariant logistic regression analyses in which the duration of diabetes, therapeutic modality of diabetes, were taken as covariates $(P<0.001$ and $P<0.001$, respectively). 
Table I

Risk factors of the study patients $(N=120)$.

\begin{tabular}{|c|c|c|c|c|c|}
\hline \multirow[t]{2}{*}{ Risk Factors } & \multicolumn{2}{|c|}{ Group I(n=60) } & \multicolumn{2}{|c|}{ Group II $(n=60)$} & \multirow[t]{2}{*}{$\mathrm{p}$ value } \\
\hline & Number & $\%$ & Number & $\%$ & \\
\hline Yes Smoking & 26 & 43.3 & 31 & 51.7 & $0.36 \mathrm{~ns}$ \\
\hline Yes Hypertension & 35 & 58.3 & 38 & 63.3 & $0.57 \mathrm{~ns}$ \\
\hline Yes Dyslipidemia & 19 & 31.7 & 14 & 23.3 & $0.31 \mathrm{~ns}$ \\
\hline \multicolumn{6}{|l|}{ Family history of premature CAD } \\
\hline Yes & 13 & 21.7 & 6 & 10.0 & $0.08 \mathrm{~ns}$ \\
\hline Mean duration of DM (yrs) & \multicolumn{2}{|c|}{$11.8 \pm 5.7$} & \multicolumn{2}{|c|}{$5.7 \pm 1.7$} & $<0.001 \mathrm{~S}$ \\
\hline No. of patients taking insulin & 22 & 36.7 & 8 & 13.3 & $0.003 \mathrm{~S}$ \\
\hline
\end{tabular}

Here,

Group I=Patients with diabetic retinopathy, Group II= Patients without diabetic retinopathy

$\mathrm{p}$ value reached from chi-square test for qualitative variables and $\mathrm{t}$-test for quantitative variables. $\mathrm{S}=\operatorname{Significant}(\mathrm{p}<0.05), \mathrm{ns}=$ Not significant $(\mathrm{p}>0.05)$

Table II

Distribution of the study patients by biochemical status $(N=120)$.

\begin{tabular}{lccc}
\hline $\begin{array}{l}\text { Biochemical } \\
\text { parameters }\end{array}$ & $\begin{array}{c}\text { Group I } \\
(\mathrm{n}=60) \\
\text { Mean } \pm \mathrm{SD}\end{array}$ & $\begin{array}{c}\text { Group II } \\
(\mathrm{n}=60) \\
\text { Mean } \pm \mathrm{SD}\end{array}$ & $\begin{array}{c}\mathrm{p} \\
\text { value }\end{array}$ \\
\hline RBS $(\mathrm{mmol} / \mathrm{L})$ & $16.1 \pm 8.6$ & $14.1 \pm 9.5$ & $0.37 \mathrm{~ns}$ \\
Serum Creatinine $(\mathrm{mg} / \mathrm{dl})$ & $1.31 \pm 0.22$ & $1.04 \pm 0.20$ & $<0.001 \mathrm{~S}$ \\
Troponin I ng/dl & $23.39 \pm 13.92$ & $21.17 \pm 12.96$ & $0.37 \mathrm{~ns}$ \\
Total Cholesterol $(\mathrm{mg} / \mathrm{dl})$ & $204.5 \pm 18.8$ & $204.3 \pm 19.7$ & $0.93 \mathrm{~ns}$ \\
LDL Cholesterol $(\mathrm{mg} / \mathrm{dl})$ & $130.6 \pm 16.6$ & $129.0 \pm 21.7$ & $0.65 \mathrm{~ns}$ \\
HDL Cholesterol $(\mathrm{mg} / \mathrm{dl})$ & $40.1 \pm 4.7$ & $39.7 \pm 5.3$ & $0.74 \mathrm{~ns}$ \\
TG (mg/dl) & $168.8 \pm 21.4$ & $0.49 \mathrm{~ns}$ \\
\hline Here, & $172.1 \pm 29.8$ \\
Group I=Patients with diabetic retinopathy, Group II= Patients without diabetic retinopathy RBS=Random blood sugar, LDL= Low density lipoprotein, \\
HDL= High density lipoprotein TG= Triglycerides \\
p value reached from unpaired t test, ns = Not significant $(\mathrm{p}>0.05), \mathrm{S}=$ Significant $(\mathrm{p}<0.05)$
\end{tabular}

Table III

Distribution of the study patients by Gensini score $(N=120)$

\begin{tabular}{|c|c|c|c|c|c|}
\hline \multirow[t]{2}{*}{ Gensini Score } & \multicolumn{2}{|c|}{ Group I $(\mathrm{n}=60)$} & \multicolumn{2}{|c|}{ Group II $(n=60)$} & \multirow[t]{2}{*}{$\mathrm{p}$ value } \\
\hline & Number & $\%$ & Number & $\%$ & \\
\hline Severe CAD ( $>36$ points) & 54 & 90.0 & 31 & 51.7 & $<0.001 S$ \\
\hline Not severe CAD (d"36points) & 6 & 10.0 & 29 & 48.3 & $<0.001 S$ \\
\hline Mean \pm SD & \multicolumn{2}{|c|}{$62.2 \pm 27.7$} & \multicolumn{2}{|c|}{$43.3 \pm 25.3$} & $<0.001 \mathrm{~S}$ \\
\hline
\end{tabular}

Here,

Group I=Patients with diabetic retinopathy, Group II= Patients without diabetic retinopathy $\mathrm{S}=$ Significant $(\mathrm{p}<0.05)$

$\mathrm{CAD}=$ Coronary artery disease

$\mathrm{p}$ value reached from chi square test of categorical approach and unpaired t-test of quantitative approach. 
Coronary angiographic findings: Comparison of coronary angiographic data showed that the patients in the DR $(+)$ group had significantly higher Gensini score with severity $(62.2 \pm 27.7$ versus $43.3 \pm 25.3, P<0.001)$ than patients in the DR (-) group (Table III).

\section{Discussion}

The results of the present study demonstrate that there is a relationship between the presence of DR severity of CAD detected by coronary angiography in patient with NSTEMI. In our study, in comparison to the patient with group II, patients of group I had significantly $(\mathrm{p}<0.002)$ higher percentage of triple vessel disease (TVD) $40 \%$ vs. $15 \%$ and double vessel disease $48.3 \%$ vs. $30 \%$. In group I there was less single vessel disease $11.7 \%$ vs. $52.3 \%$ as compared with group II and this was also statistically significant $(\mathrm{p}<0.001)$. This finding corresponds to the findings of (Norgaz et al.,2005). Saleem et al., 2017 also mentioned higher distribution of TVD, DVD and lower distribution of SVD in patients of diabetic retinopathy.

Crenshaw et al., 1997 also found more triple vessel disease (TVD) in patients with diabetic retinopathy in comparison to patients without diabetic retinopathy in non- ST elevation myocardial infarction. In our study, we saw the correlation of different stages of DR with severity of CAD. However, based on our analysis, significant correlation was found between no DR and no CAD and PDR and severe CAD. Severity of CAD increased with increase in the stage of retinopathy. Jibron MS et al., 2018 also found Severity of CAD increased with increase in the stage of retinopathy. In our study, CAD severity of the study patients was assessed by Gensini score. Moderate to severe form of CAD (Gensini score $>36$ points) was found $90 \%$ vs. $51.7 \%$ in patients of group I and group II respectively. None to mild form of CAD (Gensini score $\leq 36$ ) was found $10 \%$ vs. $48.3 \%$ in patients of group I and group II respectively. Gensini score was found to be significantly higher in diabetic retinopathy group $(62.2$ +27.7 versus $43.3+25.3$ ). In the previous study by (Norgaz et al., 2005), it was shown that the patients with diabetic retinopathy had significantly higher vessel $(2.3 \pm 0.9$ versus $1.3 \pm 1.1, \mathrm{P}<0.001)$ and severity (63.7 \pm 41.0 versus $22.6 \pm$ $14.9, \mathrm{P}<0.001)$ score than patients with no evidence of diabetic retinopathy. In (Saleem et al 2017), it was shown that the patients with diabetic retinopathy had significantly higher vessel $(2.62 \pm 0.60$ versus $1.9 \pm 1.03, \mathrm{P}<0.001)$ and severity $(103 \pm 37.17 .0$ versus $38.55 \pm 22.20, \mathrm{P}<0.001)$ score than patients with no evidence of diabetic retinopathy.

Limitations of the study: Despite utmost efforts were made during carrying out this study, there are some limitations-Sample populations was small, as purposive sampling was done so there could be a chance of selection bias, it was conducted in a single center, Prognosis of our study patients was not evaluated in this study.

\section{Conclusion:}

Presence and severity of diabetic retinopathy is associated with angiographically severe coronary artery disease in patient with non-ST elevation myocardial infarction (NSTEMI) as assessed by Gensini score which cannot explained by longer duration or inferior control of the diabetic.

\section{References}

1. Ahmed, M., Rubaiyat, K. A., Saleh, M. A. D., Chowdhury, A. W., Khuda, C. K. E., Ferdous, K. A. F., Hasan, N., Hoque, A. T. M. M., Islam, K. N. \&Amin, M. G. 'Clinical characteristics and angiographic profile of acute coronary syndrome patients in a tertiary hospital of Bangladesh', Bangladesh Heart Journal, 2018;)33(2):10-15.

2. Benziger, C. P., Moran, A. E. and Roth, G. A., 2017. The Global Burden of Cardiovascular Diseases. In: V. Fuster, R. A. Harrington, J. Narula, and Z. J. Eapen, eds. Hurst's the heart. New York: McGraw-Hill Education, 2017; 19-49.

3. Cherchi, S., Gigante, A., Spanu, M. A., Contini, P., Meloni, G., Fois, M. A et al. 'Sex-gender differences in diabetic retinopathy', In $I J D, 2020 ; 1(1): 1-10$.

4. Cheung, N., Wang, J. J., Klein, R., Couper, D. J., Sharrett, A. R. and Wong, T. Y. 'Diabetic retinopathy and the risk of coronary heart disease', Diabetes Care, 2007;30(7):1742-46.

5. Duran, J. R. and Taffet, G. 'Coronary microvascular dysfunction', The New England Journal of Medicine, 2007;356(22):2324-25

6. El Demerdash, F., Refaie, W., Allakany, R., Tantawy, S. and Dawood, E. 'Diabetic retinopathy, a predictor of coronary artery disease', The Egyptian Heart Journal, 2012;64(2):63-68.

7. Finegold, J. A., Asaria, P. and Francis, D. P. 'Mortality from ischaemic heart disease by country, region, and age, Statistics from World Health Organisation and United Nations', International Journal of Cardiology, 2013;168(2):934-45.

8. Hoque, M. M. abc of research methodology \& biostatistics: Sample size calculation, 3rd edn, Dhaka, Bangladesh Medical Book Sellers Association, 2019;146-59.

9. Islam, A. K. M. M. and Majumder, A. A. S. 'Coronary artery disease in Bangladesh', A review, Indian Heart Journal, 2013; 65(4):424-35.

10. Jibran MS, Zahid ZU, Habib SA,Shawana. Diabetic retinopathy as a predictor of severity of coronary artery disease. Khyber Med Univ J 2018;10(4):188-91.

11. Kakar, Z. A., Siddiqui, M. A., Amin, R. A. 'Prevalence and risk factors of diabetes in adult population of South Asia', Clinical Medicine and Diagnostics, 2013;3(2):18-28.

12. Norgaz, T., Hobikoglu, G., Aksu, H., Guveli, A., Aksoy, S., Ozer, O., Bolca, O. and Narin, A. 'Retinopathy is related to the angiographically detected severity and extent of coronary artery disease in patients with type 2 diabetes mellitus', International Heart Journal, 2005;46(4):639-46.

13. Pellaton, L. A., Schwitter J., Eeckhout E. 'Clinical features of myocardial infarction and myocarditis in young adults', a retrospective study, BMJ, 2012;2:e001571.

14. Resl, M., Clodi, M. 'Diabetes and cardiovascular complications', Wien Med Wochenschr, 2010;160:3-7.

15. Saunders, J., Mathewkutty, S., Drazner, M. H. and McGuire, D. K. 'Cardiomyopathy in type 2 diabetes, Update on pathophysiological mechanisms', Herz, 2008;33(3):184-90.

16. Son, J. W., Jang, E. H., Kim, M. K., Kim, I. T., Roh, Y. J., Baek, K. H.'Diabetic retinopathy is associated with subclinical atherosclerosis in newly diagnosed type 2 diabetes mellitus', 2011;91(2):253-59. 